\title{
BMS type symmetries at null-infinity and near horizon of non-extremal black holes
}

\author{
M. R. Setare ${ }^{\mathrm{a}}$, H. Adami ${ }^{\mathrm{b}}$ \\ Department of Science, University of Kurdistan, Sanandaj, Iran
}

Received: 15 September 2016 / Accepted: 29 November 2016 / Published online: 16 December 2016

(c) The Author(s) 2016. This article is published with open access at Springerlink.com

\begin{abstract}
In this paper we consider a generally covariant theory of gravity, and extend the generalized off-shell ADT current such that it becomes conserved for field dependent (asymptotically) Killing vector field. Then we define the extended off-shell ADT current and the extended off-shell ADT charge. Consequently, we define the conserved charge perturbation by integrating from the extended off-shell ADT charge over a spacelike codimension two surface. Eventually, we use the presented formalism to find the conserved charge perturbation of an asymptotically flat spacetime. The conserved charge perturbation we obtain is exactly matched with the result of Ref. (Barnich and Troessaert, 12:105 2011). These charges are as representations of the $\mathrm{BMS}_{4}$ symmetry algebra. Also, we find that the near horizon conserved charges of a non-extremal black hole with extended symmetries are the Noether charges. For this case our result is also exactly matched with that of Ref. (Donnay et al., arXiv:1607.05703 [hep-th], 2016).
\end{abstract}

\section{Introduction}

It is well known that the group of asymptotic symmetries of asymptotically flat space-times at future null infinity is the BMS group [3-5]. The BMS symmetry algebra in $n$ spacetime dimension consists of the semi-direct sum of the conformal Killing vectors of a $(n-2)$-dimension sphere acting on the ideal of infinitesimal supertranslations $[6,7]$. So, in four dimensions, the asymptotic symmetry group at null infinity of asymptotically flat space-times is not the Poincaré group. In this case, the symmetry algebra is an extension of the Poincaré algebra, in which translations are replaced by supertranslations, and contains two copies of the Virasoro algebra [1]. In contrast to 3 and 4 dimensions, in higher dimensions the supertranslations reduce to the usual translation. Thus

\footnotetext{
a e-mail: rezakord@ipm.ir

b e-mail: hamed.adami@yahoo.com
}

the asymptotical symmetry algebra of asymptotically flat space-times at the future null infinity for $n>4$ dimensions is just the Poincaré algebra [7-9]. The infinite-dimensional supertranslation subgroup of $\mathrm{BMS}_{4}$ generates arbitrary angle dependent translations of retarded time. Ashtekar has investigated the implications of the supertranslations in the context of asymptotic quantization [10,11]. Recently, Donnay et al. [12], have shown that the asymptotic symmetries close to the horizon of the non-extremal black hole solution of the three-dimensional Einstein gravity in the presence of a negative cosmological term, are generated by an extension of supertranslations. They have shown that for a special choice of boundary conditions, the near region to the horizon of a stationary black hole presents a generalization of supertranslation, including a semidirect sum with superrotations, represented by Virasoro algebra (see also [13]). More recently, we have studied the behaviors and algebras of the symmetries and conserved charges near the horizon of the nonextremal black holes in the context of the so-called Generalized Minimal Massive Gravity [14], proposed in Ref. [15]. In an interesting paper [16] Strominger has studied the $\mathrm{BMS}_{4}$ invariance of gravitational scattering. He has discussed that in a finite neighborhood of the Minkowski vacuum, classical gravitational scattering is in fact BMS-invariant. He has demonstrated BMS invariance of the S-matrix, and has shown that the supertranslation invariance implies energy conservation at every angle. In extension of AdS/CFT correspondence to the flat space holography, the BMS algebra has been investigated very much in recent years [1,6, 17-34]. Since the $\mathrm{BMS}_{4}$ algebra is an extension of the Poincaré algebra, the asymptotically flat space-time in four dimensions is dual to an extended conformal field theory. The $\mathrm{BMS}_{4}$ charge algebra has been studied in [1]. The authors of [1] have used the covariant approach in order to obtain surface charges and their algebra (see also [35-37]). The $\mathrm{BMS}_{4}$ Surface-charge algebra on the null infinity of asymptotically flat spacetime has been investigated via the Hamiltonian formalism 
in the reference [38]. The $\mathrm{BMS}_{4}$ group leads to the conserved charges, a part of these conserved charges are associated to the Poincaré group, another part is an infinite number of supermomentum charge associated with supertranslations. In Refs. [1,7,24] Barnich and Troessaert have discussed the vector fields called "superrotations", which correspond to the infinitesimal symmetries but cannot be exponentiated to lead smooth finite diffeomorphisms. Recently, Flanagan and Nichols have computed the superrotation charges, and have shown that these charges which are called "super center-ofmass" by them are in general finite [39].

In this paper we are going to show that the BMS symmetries appear at null infinity of asymptotically flat fourdimensional space-times. What we find in this case is exactly matched with the result of Ref. [1], but here, we obtain this result by a different approach. On the other hand, the near horizon geometry of non-extremal black hole solutions of a generally covariant theory of gravity exhibits an infinitedimensional symmetry which is not exactly $\mathrm{BMS}_{4}$ [2]. In fact, at the horizon we find the so-called $\mathrm{BMS}_{4}^{H}$ algebra provided in reference [2].

In this paper at first we consider a generally covariant theory of gravity in $D$ dimensions. Then we try to find the quasilocal conserved charges corresponding to the field-dependent Killing vector fields. We assume that the diffeomorphism generator $\xi$ depends on the dynamical fields which appear in the metric. Then by using the ADT method [40-42], developed in [43-45] (for the recent works see [46-48]), we obtain the extended off-shell ADT current. Afterward, we define the perturbation of the conserved charge by integrating of the extended off-shell ADT charge over a space-like codimension two surface. As an application of this method for computation of conserved charges, when the Killing vector fields are dependent on the dynamical fields in the metric, and in the direction of our aims, we consider an asymptotically flat line element where the components of the metric are functions of the coordinates. Our result for a conserved charge perturbation is exactly matched with that of Ref. [1]. These charges are as representations of the $\mathrm{BMS}_{4}$ symmetry algebra. Very recently the authors of [2] have shown that the non-extremal black holes in four-dimensional general relativity exhibit an infinite-dimensional symmetry in their near horizon region. The algebra they have found contains two sets of supertranslations currents, besides, it contains two sets of Virasoro currents which are in semi-direct sum with the supertranslations. Due to the presence of two sets of supertranslation currents, this algebra is not exactly the $\mathrm{BMS}_{4}$ symmetry algebra which includes two copies of Virasoro algebra and one set of supertranslations. In Sect. 3 we try to find the expression of the conserved charges associated to the near horizon symmetry of the non-extremal black hole solution of general relativity in four dimensions by the Noether method. Our result for this case is also exactly matched with that of Ref. [2].

\section{Quasi-local conserved charges correspond to the field dependent Killing vector fields}

The action of a generally covariant theory of gravity in $D$ dimensions is given by

$I=\frac{1}{16 \pi G} \int \mathrm{d}^{D} x \sqrt{-g} \mathcal{L}$,

where $G$ is the gravitational constant and $\mathcal{L}=\mathcal{L}\left(g_{\mu \nu}, R, R_{\mu \nu}\right.$ $\left.R^{\mu \nu}, \ldots\right)$ is the Lagrangian density of a generally covariant theory of gravity. By varying Eq. (1) with respect to metric $g_{\mu \nu}$ we have

$\delta(\sqrt{-g} \mathcal{L})=\sqrt{-g} \mathcal{E}^{\mu v} \delta g_{\mu \nu}+\partial_{\mu} \Theta^{\mu}(g ; \delta g)$,

where $\mathcal{E}^{\mu \nu}=0$ are the equations of motions and $\Theta^{\mu}(g ; \delta g)$ is the surface term. The variation of the metric under a diffeomorphism generated by the vector field $\xi^{\mu}$ is $\delta_{\xi} g_{\mu \nu}=$ $\nabla_{\mu} \xi_{\nu}+\nabla_{\nu} \xi_{\mu}$. By supposing that the variation in Eq. (2) is due to a diffeomorphism generated by the vector field $\xi^{\mu}$ we find that

$\delta_{\xi}(\sqrt{-g} \mathcal{L})=2 \sqrt{-g} \mathcal{E}^{\mu \nu} \nabla_{\mu} \xi_{v}+\partial_{\mu} \Theta^{\mu}\left(g ; \delta_{\xi} g\right)$.

It is known that $\sqrt{-g}$ is a scalar density of weight +1 and the Bianchi identity is given by $\nabla_{\nu} \mathcal{E}^{\mu \nu}=0$, then Eq. (3) can be written as

$\partial_{\mu} J^{\mu}=0$,

where $J^{\mu}=J^{\mu}(g ; \xi)$ is an off-shell conserved current and it is given by

$J^{\mu}=\Theta^{\mu}\left(g ; \delta_{\xi} g\right)-\sqrt{-g} \mathcal{L} \xi^{\mu}+2 \sqrt{-g} \mathcal{E}^{\mu v} \xi_{\nu}$.

By virtue of the Poincaré lemma, one can write

$J^{\mu}(g ; \xi)=\partial_{\nu} K^{\nu \mu}(g ; \xi)$,

where $K^{\mu \nu}=-K^{v \mu}$. Now, we assume that the diffeomorphism generator $\xi$ depends on the dynamical fields which are appear in the metric. By varying Eq. (5) with respect to the dynamical fields we find that

$$
\begin{aligned}
\partial_{v} & \left(\hat{\delta} K^{v \mu}(g ; \xi)-K^{v \mu}(g ; \hat{\delta} \xi)-2 \xi^{[v} \Theta^{\mu]}(g ; \hat{\delta} g)\right) \\
= & \hat{\delta} \Theta^{\mu}(g ; \delta \xi g)-\delta_{\xi} \Theta^{\mu}(g ; \hat{\delta} g)-\Theta^{\mu}\left(g ; \delta_{\hat{\delta} \xi} g\right) \\
& +2 \sqrt{-g}\left(\hat{\delta} \mathcal{E}^{\mu \nu} \xi_{v}+\mathcal{E}^{\mu \nu} \hat{\delta} g_{v \lambda} \xi^{\lambda}-\frac{1}{2} \xi^{\mu} \mathcal{E}^{\alpha \beta} \hat{\delta} g_{\alpha \beta}\right. \\
& \left.+\frac{1}{2} g^{\alpha \beta} \hat{\delta} g_{\alpha \beta} \mathcal{E}^{\mu \nu} \xi_{v}\right)
\end{aligned}
$$

where $\hat{\delta}$ denotes variation with respect to the dynamical fields. The off-shell ADT current is defined as [43-45]

$$
\begin{gathered}
\sqrt{-g} \mathcal{J}_{\mathrm{ADT}}^{\mu}(g, \delta g ; \xi)=\delta \mathcal{E}^{\mu v} \xi_{v}+\mathcal{E}^{\mu v} \delta g_{\nu \lambda} \xi^{\lambda} \\
-\frac{1}{2} \xi^{\mu} \mathcal{E}^{\alpha \beta} \delta g_{\alpha \beta}+\frac{1}{2} g^{\alpha \beta} \delta g_{\alpha \beta} \mathcal{E}^{\mu v} \xi_{v} .
\end{gathered}
$$


The off-shell ADT current $\mathcal{J}_{\mathrm{ADT}}^{\mu}(g ; \delta g)$ is conserved offshell for arbitrary field-independent Killing vector field which is admitted by the spacetime everywhere. Also, the symplectic current define as an antisymmetric bilinear map on perturbations [49]

$$
\begin{aligned}
& \omega^{\mu}\left(g ; \delta_{1} g, \delta_{2} g\right)=\delta_{1} \Theta^{\mu}\left(g ; \delta_{2} g\right) \\
& \quad-\delta_{2} \Theta^{\mu}\left(g ; \delta_{1} g\right)-\Theta^{\mu}\left(g ;\left[\delta_{1}, \delta_{2}\right] g\right) .
\end{aligned}
$$

The above expression for the symplectic current reduces to the Lee-Wald one [50-53], namely $\omega_{\mathrm{LW}}^{\mu}=\delta_{1} \Theta^{\mu}\left(g ; \delta_{2} g\right)-$ $\delta_{2} \Theta^{\mu}\left(g ; \delta_{1} g\right)$, when two variations $\delta_{1}$ and $\delta_{2}$ are commute, i.e. $\left[\delta_{1}, \delta_{2}\right] g=0$. The symplectic current $(9)$ is conserved on-shell and it gives us conserved charges correspond to asymptotically field-independent Killing vectors. It should be noted that for the case in which $\xi$ is field-dependent we have $\left[\hat{\delta}, \delta_{\xi}\right]=\delta_{\hat{\delta} \xi}$, then Eq. (9) becomes

$$
\begin{aligned}
& \omega^{\mu}\left(g ; \hat{\delta} g, \delta_{\xi} g\right)=\hat{\delta} \Theta^{\mu}\left(g ; \delta_{\xi} g\right) \\
& -\delta_{\xi} \Theta^{\mu}(g ; \hat{\delta} g)-\Theta^{\mu}\left(g ; \delta_{\hat{\delta} \xi} g\right) .
\end{aligned}
$$

It is easy to see that Eq. (10) will be reduced to the Lee-Wald symplectic current when $\xi$ is field-independent, i.e. $\hat{\delta} \xi=0$. In Ref. [54], the authors have generalized the off-shell ADT current as follows:

$$
\begin{aligned}
& \mathcal{J}_{\mathrm{GADT}}^{\mu}(g, \delta g ; \xi)=\mathcal{J}_{\mathrm{ADT}}^{\mu}(g, \delta g ; \xi) \\
& +\frac{1}{2 \sqrt{-g}} \omega_{\mathrm{LW}}^{\mu}\left(g ; \delta g, \delta_{\xi} g\right),
\end{aligned}
$$

this current is conserved off-shell for the asymptotically fieldindependent Killing vector fields as well as field-independent Killing vector fields admitted by spacetime everywhere.

For the case in which $\xi$ depends on the dynamical fields, it seems to be sensible replacing $\delta$ and the Lee-Wald symplectic current by $\hat{\delta}$ and $\omega^{\mu}\left(g ; \hat{\delta} g, \delta_{\xi} g\right)$ in Eq. (11), respectively. Thus, we can define the extended off-shell ADT current as

$\mathfrak{J}_{\mathrm{ADT}}^{\mu}(g, \hat{\delta} g ; \xi)=\mathcal{J}_{\mathrm{ADT}}^{\mu}(g, \hat{\delta} g ; \xi)+\frac{1}{2 \sqrt{-g}} \omega^{\mu}(g ; \hat{\delta} g, \delta \xi g)$.

It is clear that the extended off-shell ADT current $\mathfrak{J}_{\mathrm{ADT}}^{\mu}$ is conserved off-shell for asymptotically field dependent Killing vector fields as well as field-dependent Killing vector fields admitted by spacetime everywhere. By considering Eq. (12), one can rewrite Eq. (7) as follows:

$\sqrt{-g} \mathfrak{J}_{\mathrm{ADT}}^{\mu}(g, \hat{\delta} g ; \xi)=\partial_{\nu}\left[\sqrt{-g} \mathcal{Q}_{\mathrm{ADT}}^{\nu \mu}(g, \hat{\delta} g ; \xi)\right]$,

where $\mathcal{Q}_{\mathrm{ADT}}^{\mu \nu}(g, \hat{\delta} g ; \xi)$ is defined as the extended off-shell ADT charge and it is given by

$$
\begin{gathered}
\sqrt{-g} \mathcal{Q}_{\mathrm{ADT}}^{\mu \nu}(g, \hat{\delta} g ; \xi)=\frac{1}{2} \hat{\delta} K^{\mu \nu}(g ; \xi) \\
-\frac{1}{2} K^{\mu \nu}(g ; \hat{\delta} \xi)-\xi^{[\mu} \Theta^{\nu]}(g ; \hat{\delta} g) .
\end{gathered}
$$

By substituting $K^{\mu \nu}=\sqrt{-g} \tilde{K}^{\mu \nu}$ and $\Theta^{\mu}=\sqrt{-g} \tilde{\Theta}^{\mu}$ into Eq. (14) we have

$$
\begin{aligned}
\mathcal{Q}_{\mathrm{ADT}}^{\mu \nu}(g, \hat{\delta} g ; \xi)= & \frac{1}{2} \hat{\delta} \tilde{K}^{\mu \nu}(g ; \xi)+\frac{1}{4} g^{\alpha \beta} \hat{\delta} g_{\alpha \beta} \tilde{K}^{\mu \nu}(g ; \xi) \\
& -\frac{1}{2} \tilde{K}^{\mu \nu}(g ; \hat{\delta} \xi)-\xi^{[\mu} \tilde{\Theta}^{\nu]}(g ; \hat{\delta} g) .
\end{aligned}
$$

The above expression for the extended off-shell ADT charge is reduced to the generalized ADT charge [54] when $\xi$ is fieldindependent, i.e. $\hat{\delta} \xi=0$. Now, we can define the perturbation of the conserved charge by integrating from the extended offshell ADT charge over a spacelike codimension two surface

$\hat{\delta} Q(\xi)=\frac{1}{16 \pi G} \int_{\Sigma}\left(d^{D-2} x\right)_{\mu \nu} \sqrt{-g} \mathcal{Q}_{\mathrm{ADT}}^{\mu \nu}(g, \hat{\delta} g ; \xi)$,

where

$\left(d^{D-2} x\right)_{\mu \nu}=\frac{1}{2(D-2) !} \varepsilon_{\mu \nu \alpha_{1} \cdots \alpha_{D-2}} \mathrm{~d} x^{\alpha_{1}} \cdots \mathrm{d} x^{\alpha_{D-2}}$.

The charge defined by Eq. (16) is conserved off-shell for the asymptotically field-dependent Killing vector fields as well as field-dependent Killing vector fields admitted by spacetime everywhere.

As we mentioned earlier, the Lagrangian of a generally covariant theory of gravity is given by $\mathcal{L}=\mathcal{L}\left(g_{\mu \nu}, R, R_{\mu \nu}\right.$ $\left.R^{\mu \nu}, \ldots\right)$, so we have the following expressions for $\tilde{K}^{\mu \nu}$ and $\tilde{\Theta}^{\mu}[45]$ :

$$
\begin{aligned}
& \tilde{K}^{\mu \nu}(g ; \xi)=2 P^{\mu \nu \alpha \beta} \nabla_{\alpha} \xi_{\beta}-4 \xi_{\beta} \nabla_{\alpha} P^{\mu \nu \alpha \beta}, \\
& \tilde{\Theta}^{\mu}(g ; \hat{\delta} g)=2\left(P^{\mu \alpha \beta \nu} \nabla_{\nu} \hat{\delta} g_{\alpha \beta}-\hat{\delta} g_{\alpha \beta} \nabla_{\nu} P^{\mu \alpha \beta \nu}\right),
\end{aligned}
$$

where $P^{\mu \nu \alpha \beta}=\partial \mathcal{L} / \partial R_{\mu \nu \alpha \beta}$. For Einstein gravity, we have

$P^{\alpha \mu \beta \nu}=\frac{1}{2}\left(g^{\mu \nu} g^{\alpha \beta}-g^{\alpha \nu} g^{\mu \beta}\right)$,

therefore in this case Eq. (18) will be reduced to

$$
\begin{aligned}
\tilde{K}^{\mu \nu}(g ; \xi) & =2 \nabla^{[\mu} \xi^{\nu]}, \\
\tilde{\Theta}^{\mu}(g ; \hat{\delta} g) & =\nabla^{\alpha}\left(g^{\mu \beta} \hat{\delta} g_{\alpha \beta}\right)-\nabla^{\mu}\left(g^{\alpha \beta} \hat{\delta} g_{\alpha \beta}\right) .
\end{aligned}
$$

By substituting Eq. (20) into Eq. (15) we find that ${ }^{1}$

$$
\begin{aligned}
\mathcal{Q}_{\mathrm{ADT}}^{\mu \nu}(g, \hat{\delta} g ; \xi) & \\
= & -h^{\lambda[\mu} \nabla_{\lambda} \xi^{\nu]}+\xi^{\lambda} \nabla^{[\mu} h_{\lambda}^{\nu]}+\frac{1}{2} h \nabla^{[\mu} \xi^{\nu]} \\
& -\xi^{[\mu} \nabla_{\lambda} h^{\nu] \lambda}+\xi^{[\mu} \nabla^{\nu]} h,
\end{aligned}
$$

where $h_{\mu \nu}=\hat{\delta} g_{\mu \nu}$. Although this formula is independent of $\hat{\delta} \xi$, we have shown that this formula is valid for the case in which $\xi$ is field-dependent.

\footnotetext{
${ }^{1}$ It is easy to see that the Abbott-Deser formula (see Eq. (2.1) of [1]) has two more terms than Eq. (21).
} 


\subsection{An example}

Let $x^{\mu}=(u, r, \theta, \phi)$ and $A, B, \cdots=2,3$. We consider an asymptotically flat spacetime presented in [25]. The line element of an asymptotically flat spacetime can be written in the following form $[5,25]$ :

$$
\begin{aligned}
\mathrm{d} s^{2}= & e^{2 X} \frac{V}{r} \mathrm{~d} u^{2}-2 e^{2 X} \mathrm{~d} u \mathrm{~d} r \\
& +g_{A B}\left(\mathrm{~d} x^{A}-U^{A} \mathrm{~d} u\right)\left(\mathrm{d} x^{B}-U^{B} \mathrm{~d} u\right),
\end{aligned}
$$

where $X, V$, and $g_{A B}\left(\operatorname{det} g_{A B}\right)^{-1 / 2}$ are six functions of the coordinates. Also, we assume that $g^{A C} g_{C B}=\delta_{B}^{A}$ and we impose the following gauge conditions:

$g_{r r}=0, \quad g_{r A}=0$,

$\partial_{r}\left(r^{-4} \operatorname{det} g_{A B}\right)=0$.

The line element (22) solves the Einstein field equations when $g_{A B}, X, V$ and $U^{A}$ are given as

$$
g_{A B}=r^{2} \bar{\gamma}_{A B}+r \bar{C}_{A B}+\bar{D}_{A B}+\frac{1}{4} \bar{\gamma}_{A B} \bar{C}_{D}^{C} \bar{C}_{C}^{D}+\mathcal{O}\left(r^{-\varepsilon}\right),
$$

$$
\begin{aligned}
X=- & \frac{1}{32 r^{2}} \bar{C}_{B}^{A} \bar{C}_{A}^{B}-\frac{1}{12 r^{3}} \bar{C}_{B}^{A} \bar{D}_{A}^{B}+\mathcal{O}\left(r^{-3-\varepsilon}\right), \\
\frac{V}{r}= & -\frac{1}{2} \bar{R}+\frac{2 M}{r}+\mathcal{O}\left(r^{-1-\varepsilon}\right), \\
U^{A}= & -\frac{1}{2 r^{2}} \bar{\nabla}_{B} \bar{C}^{A B} \\
& -\frac{2}{3 r^{3}}\left[\left(\ln r+\frac{1}{3}\right) \bar{\nabla}_{B} \bar{D}^{A B}\right. \\
& \left.-\frac{1}{2} \bar{C}_{B}^{A} \bar{\nabla}_{C} \bar{C}^{C B}+\bar{N}^{A}\right]+\mathcal{O}\left(r^{-2-\varepsilon}\right),
\end{aligned}
$$

where $\bar{\gamma}^{A C} \bar{\gamma}_{C B}=\delta_{B}^{A}, \bar{\gamma}_{A B} d x^{A} d x^{B}=e^{2 \varphi\left(x^{C}\right)}\left(d \theta^{2}+\right.$ $\sin ^{2} \theta d \phi^{2}$ ) and indices on $\bar{C}_{A B}$ and $\bar{D}_{A B}$ are raised with $\bar{\gamma}^{A B}$. Also, $\bar{C}_{C}^{C}=\bar{D}_{C}^{C}=\partial_{u} \bar{D}_{A B}=0 . \bar{\nabla}_{A}$ is the covariant derivative associated with $\bar{\gamma}_{A B}$ and $\bar{R}$ is the scalar curvature of $\bar{\nabla}_{A}$. In Eq. (27), $M=M\left(u, x^{A}\right)$ is the mass aspect and, in Eq. (28), $\bar{N}^{A}=\bar{N}^{A}\left(u, x^{B}\right)$ is the angular momentum aspect. We should mention that $\bar{C}_{A B}, \bar{D}_{A B}, M$, and $\bar{N}^{A}$ are dynamical fields. The metric under transformations generated by $\xi$ transforms as $\delta_{\xi} g_{\mu \nu}=\mathfrak{\xi}_{\xi} g_{\mu \nu}$. The variation generated by the following vector field preserves the fall-off conditions (25)-(28):

$$
\begin{aligned}
& \xi^{u}=f, \\
& \xi^{r}=-\frac{r}{2}\left(\bar{\nabla}_{A} \xi^{A}-f_{, B} U^{B}\right), \\
& \xi^{A}=Y^{A}+I^{A} ; \quad I^{A}=-f_{, B} \int_{r}^{\infty} d r^{\prime}\left(e^{2 X} g^{A B}\right),
\end{aligned}
$$

where $f=e^{\varphi} T+\frac{1}{2} u \psi, Y^{A}=Y^{A}\left(x^{B}\right), T=T\left(x^{B}\right)$ and $\psi=\bar{\nabla}_{A} Y^{A}$, where $Y^{A}$ is a conformal Killing vector of $\bar{\gamma}_{A B}$. Preserving the boundary conditions means that the metric $g_{\mu \nu}(\Phi)$ is mapped into $g_{\mu \nu}\left(\Phi+\delta_{\xi} \Phi\right)$ by transforms generated by $\xi$, where $\Phi$ is the collection of dynamical fields. The change of dynamical fields $\delta \xi \Phi$ under a transformation generated by $\xi$ is given in Ref. [1].

Now, we take codimension two surface $\Sigma$ to be a $(u, r)$ constant surface. Thus, the $r u$ component of the extended ADT charge is important. We can rewrite the $r u$ component of the extended ADT charge (21) as

$$
\begin{aligned}
2 \mathcal{Q}_{\mathrm{ADT}}^{r u}= & \xi^{r}\left[\nabla^{r} h_{r}^{u}+\nabla^{u} h-\nabla^{u} h_{r}^{r}-\nabla_{\lambda} h^{u \lambda}\right] \\
& +\xi^{u}\left[\nabla^{r} h_{u}^{u}-\nabla^{r} h-\nabla^{u} h_{u}^{r}+\nabla_{\lambda} h^{r \lambda}\right] \\
& +\xi^{A}\left[\nabla^{r} h_{A}^{u}-\nabla^{u} h_{A}^{r}\right]+\frac{1}{2} h\left(\nabla^{r} \xi^{u}-\nabla^{u} \xi^{r}\right) \\
& +h^{\lambda u} \nabla_{\lambda} \xi^{r}-h^{\lambda r} \nabla_{\lambda} \xi^{u} .
\end{aligned}
$$

Two last terms in the right hand side of Eq. (30) can be simplified as

$$
\begin{aligned}
\frac{1}{2} h & \left(\nabla^{r} \xi^{u}-\nabla^{u} \xi^{r}\right)+h^{\lambda u} \nabla_{\lambda} \xi^{r}-h^{\lambda r} \nabla_{\lambda} \xi^{u} \\
= & \left(\frac{1}{2} h g^{r u}-h^{r u}\right)\left(\nabla_{u} \xi^{u}-\nabla_{r} \xi^{r}\right) \\
& +\left(\frac{1}{2} h g^{r A}-h^{r A}\right) \nabla_{A} \xi^{u}
\end{aligned}
$$

where we have used the equation $\nabla_{r} \xi^{u}=0$. Since $h^{\mu \nu}=$ $g^{\mu \alpha} g^{\nu \beta} \hat{\delta} g_{\alpha \beta}=-\hat{\delta} g^{\mu \nu}$, then by considering line element (22), we find that

$$
\begin{aligned}
& \frac{1}{2} h\left(\nabla^{r} \xi^{u}-\nabla^{u} \xi^{r}\right)+h^{\lambda u} \nabla_{\lambda} \xi^{r}-h^{\lambda r} \nabla_{\lambda} \xi^{u} \\
& =-e^{-2 X} \hat{\delta} U^{A} \nabla_{A} \xi^{u} .
\end{aligned}
$$

By substituting Eqs. (25) and (26) into Eq. (29), we have

$\xi^{A}=Y^{A}-\frac{1}{r} \bar{\nabla}^{A} f+\frac{1}{2 r^{2}} \bar{C}^{A B} \bar{\nabla}_{B} f+\mathcal{O}\left(r^{-3}\right)$,

and by some calculations one can show that

$\nabla_{A} \xi^{u}=r Y_{A}+\frac{1}{2} \bar{C}_{A B} Y^{B}+\mathcal{O}\left(r^{-1}\right)$,

$\xi^{r}=-\frac{1}{2} r \psi+\frac{1}{2} \bar{\square} f+\mathcal{O}\left(r^{-1}\right)$.

By substituting Eqs. (34) and (28) into Eq. (32) we find that

$$
\begin{aligned}
& r^{2} e^{2 X}\left[\frac{1}{2} h\left(\nabla^{r} \xi^{u}-\nabla^{u} \xi^{r}\right)+h^{\lambda u} \nabla_{\lambda} \xi^{r}-h^{\lambda r} \nabla_{\lambda} \xi^{u}\right] \\
& =\frac{1}{2} r Y_{A} \bar{\nabla}_{B} \hat{\delta} \bar{C}^{A B} \\
& +\left[\frac{2}{3}\left(\ln r+\frac{1}{3}\right) Y_{A} \bar{\nabla}_{B} \hat{\delta} \bar{D}^{A B}-\frac{1}{3} Y_{A} \hat{\delta}\left(\bar{C}_{B}^{A} \bar{\nabla}_{C} \bar{C}^{B C}\right)\right.
\end{aligned}
$$




$$
\begin{aligned}
& \left.+\frac{2}{3} Y_{A} \hat{\delta} \bar{N}^{A}\right] \\
& +\frac{1}{4} \bar{\nabla}_{B} \hat{\delta} \bar{C}^{A B} \bar{C}_{A C} Y^{C}+\mathcal{O}\left(r^{-1}\right) .
\end{aligned}
$$

Also, we have [1]

$$
\begin{aligned}
& \nabla^{r} h_{r}^{u}+\nabla^{u} h-\nabla^{u} h_{r}^{r}-\nabla_{\lambda} h^{u \lambda} \\
& =\frac{1}{4 r^{3}} \bar{C}^{A B} \hat{\delta} \bar{C}_{A B}+\mathcal{O}\left(r^{-3-\varepsilon}\right) \\
& \nabla^{r} h_{u}^{u}-\nabla^{r} h-\nabla^{u} h_{u}^{r}+\nabla_{\lambda} h^{r \lambda} \\
& =\frac{1}{r^{2}}\left[4 \hat{\delta} M-\frac{1}{2} \bar{\nabla}_{A} \bar{\nabla}_{B} \hat{\delta} \bar{C}^{A B}+\frac{1}{2} \partial_{u} \bar{C}^{A B} \hat{\delta} \bar{C}_{A B}\right] \\
& \quad+\mathcal{O}\left(r^{-2-\varepsilon}\right), \\
& \nabla^{r} h_{A}^{u}-\nabla^{u} h_{A}^{r}=\frac{1}{2 r} \bar{\nabla}_{B} \hat{\delta} \bar{C}_{A}^{B}+\frac{2}{3 r^{2}}\left(2 \ln r-\frac{1}{3}\right) \bar{\nabla}_{B} \hat{\delta} \bar{D}_{A}^{B} \\
& \quad+\frac{1}{r^{2}}\left[\frac{4}{3} \hat{\delta} \bar{N}_{A}+\frac{1}{3} \hat{\delta}\left(\bar{C}_{A B} \bar{\nabla}_{C} \bar{C}^{B C}\right)\right. \\
& \left.\quad-\frac{1}{4} \bar{C}_{A B} \bar{\nabla}_{C} \hat{\delta} \bar{C}^{B C}\right] \\
& \quad+\mathcal{O}\left(r^{-2-\varepsilon}\right) .
\end{aligned}
$$

As we mentioned earlier, we take the codimension two surface $\Sigma$ to be a $(u, r)$-constant surface so Eq. (16) becomes

$$
\not{\delta} Q(\xi)=\frac{1}{8 \pi G} \int_{2 \text {-sphere }} d^{2} \Omega\left(r^{2} e^{2 X} \mathcal{Q}_{\mathrm{ADT}}^{r u}\right),
$$

where

$d^{2} \Omega=e^{2 \varphi} \sin \theta d \theta d \phi$.

The symbol $\not f$ emphasizes that the perturbation of conserved charge may be non-integrable. By substituting Eqs. (36)-(39) into Eq. (40) we have

$$
\begin{aligned}
& \not \delta Q(\xi) \\
& =\frac{1}{16 \pi G} \int_{2 \text {-sphere }} d^{2} \Omega\left\{r Y_{A} \bar{\nabla}_{B} \hat{\delta} \bar{C}^{A B}-\frac{1}{2} \bar{\nabla}_{A} f \bar{\nabla}_{B} \hat{\delta} \bar{C}^{A B}\right. \\
& -\frac{1}{8} \psi \bar{C}^{A B} \hat{\delta} \bar{C}_{A B}+2 \ln r Y_{A} \bar{\nabla}_{B} \hat{\delta} \bar{D}^{A B} \\
& +2 Y_{A} \hat{\delta} \bar{N}^{A}+4 f \hat{\delta} M-\frac{1}{2} f \bar{\nabla}_{A} \bar{\nabla}_{B} \hat{\delta} \bar{C}^{A B} \\
& \left.+\frac{1}{2} f \partial_{u} \bar{C}^{A B} \hat{\delta} \bar{C}_{A B}\right\} .
\end{aligned}
$$

Using the conformal Killing equation for the $Y^{A}$, i.e. $\bar{\nabla}_{(A} Y_{B)}=\frac{1}{2} \bar{\gamma}_{A B} \bar{\nabla}_{C} Y^{C}$, and integrations by parts, Eq. (42) can be simplified to

$$
\begin{aligned}
\not \partial Q(\xi)= & \frac{1}{16 \pi G} \hat{\delta} \int_{2 \text {-sphere }} d^{2} \Omega \\
& \times\left\{4 f M+Y^{A}\left[2 \bar{N}_{A}+\frac{1}{16} \partial_{A}\left(\bar{C}^{B C} \bar{C}_{B C}\right)\right]\right\} \\
& +\frac{1}{16 \pi G} \int_{2 \text {-sphere }} d^{2} \Omega\left[\frac{1}{2} f \partial_{u} \bar{C}^{A B} \hat{\delta} \bar{C}_{A B}\right] .
\end{aligned}
$$

In the right hand side of Eq. (43), the first term is the integrable part of the surface charge and the second term is the non-integrable part. The conserved charge perturbation (43) which we found in this way is exactly matched with that of Ref. [1]. Here we have obtained this result by a different approach. As has been discussed in [1] these charges behave as representations of the $\mathrm{BMS}_{4}$ symmetry algebra.

\section{Near horizon conserved charges of non-extremal black holes as Noether charges}

Consider near horizon metric of a non-extremal black hole in the Eddington-Finkelstein coordinates. Let $v$ and $\rho$ are the advanced time and radial coordinate, respectively. We suppose that the event horizon (which is a null surface) located at $\rho=0$ and it is a non-expanding surface. Following $[2,55,56]$, the components of the metric close to the near horizon region behave like

$$
g_{\mu \nu}=\left(\begin{array}{ccc}
-2 \kappa \rho+\mathcal{O}\left(\rho^{2}\right) & 1 & \rho N_{B}+\mathcal{O}\left(\rho^{2}\right) \\
1 & 0 & 0 \\
\rho N_{A}+\mathcal{O}\left(\rho^{2}\right) & 0 & \Omega_{A B}+\rho \lambda_{A B}+\mathcal{O}\left(\rho^{2}\right)
\end{array}\right),
$$

where $\kappa, N_{A}, \Omega_{A B}$ and $\lambda_{A B}$ are functions of $\left(v, x^{A}\right)$. As before, we assume that $x^{A}=(\theta, \phi)$. The inverse of the metric (45) is given by

$g^{\mu \nu}=\left(\begin{array}{ccc}0 & 1 & 0 \\ 1 & 2 \kappa \rho+\mathcal{O}\left(\rho^{2}\right) & -\rho N^{B}+\mathcal{O}\left(\rho^{2}\right) \\ 0 & -\rho N^{A}+\mathcal{O}\left(\rho^{2}\right) & \Omega^{A B}-\rho \lambda^{A B}+\mathcal{O}\left(\rho^{2}\right)\end{array}\right)$,

where $\Omega^{A B}$ is the inverse of $\Omega_{A B}$ and, in this section, $A, B, \ldots$ indices are raised with $\Omega^{A B}$. By some calculations, one can show that the metric connections corresponding to the metric (44) are

$$
\begin{aligned}
& \Gamma_{\rho \rho}^{\lambda}=0, \Gamma_{\lambda \rho}^{v}=0, \Gamma_{A \rho}^{\rho}=\frac{1}{2} N_{A}+\mathcal{O}(\rho), \\
& \Gamma_{B \rho}^{A}=\frac{1}{2} \lambda_{B}^{A}+\mathcal{O}(\rho), \\
& \Gamma_{A B}^{v}=-\frac{1}{2} \lambda_{A B}+\mathcal{O}(\rho), \quad \Gamma_{B C}^{A}=\tilde{\Gamma}_{B C}^{A}+\mathcal{O}(\rho), \\
& \Gamma_{\rho v}^{A}=\frac{1}{2} N^{A}+\mathcal{O}(\rho), \\
& \Gamma_{v A}^{v}=-\frac{1}{2} N_{A}, \quad \Gamma_{\rho v}^{\rho}=-\kappa+\mathcal{O}(\rho),
\end{aligned}
$$




$$
\begin{aligned}
& \Gamma_{B v}^{A}=\frac{1}{2} \Omega^{A C} \Omega_{C B, v}+\mathcal{O}(\rho), \\
& \Gamma_{v v}^{v}=\kappa, \quad \Gamma_{A B}^{\rho}=-\frac{1}{2} \Omega_{A B, v}+\mathcal{O}(\rho), \\
& \Gamma_{A v}^{\rho}=\mathcal{O}(\rho), \quad \Gamma_{v v}^{\rho}=\mathcal{O}(\rho), \\
& \Gamma_{v v}^{A}=\mathcal{O}(\rho),
\end{aligned}
$$

where $\tilde{\Gamma}_{B C}^{A}$ is the connection associated to $\Omega_{A B}$.

The variation generated by the following vector field preserves the fall-off conditions (44):

$\chi^{v}=f$,

$\chi^{\rho}=-\rho \partial_{v} f+\frac{1}{2} \rho^{2} N^{A} \partial_{A} f+\mathcal{O}\left(\rho^{3}\right)$,

$\chi^{A}=Y^{A}+\rho \partial^{A} f+\frac{1}{2} \rho^{2} \lambda^{A B} \partial_{B} f+\mathcal{O}\left(\rho^{3}\right)$,

where $f=f\left(v, x^{A}\right)$ and $Y^{A}=Y^{A}\left(x^{B}\right)$ [2]. Here, as we mentioned in the previous section, the metric under transformation generated by $\chi$ transforms as $\delta_{\chi} g_{\mu \nu}=£_{\chi} g_{\mu \nu}$. In this section, we consider the gauge conditions $g_{\rho \rho}=g_{\rho A}=0$ and $g_{\rho v}=1$. Due to these gauge conditions the $v$ component of $\chi$ has been fixed exactly. Also, the fall-off conditions (44) are considered up to $\mathcal{O}\left(\rho^{2}\right)$ so to preserve these boundary conditions under transformations generated by $\chi$, we need to consider $\chi^{\rho}$ and $\chi^{A}$ up to $\mathcal{O}\left(\rho^{3}\right)$ (see Ref. [2]).

In Sect. 2, we have showed that $J^{\mu}$ is a Noether current density which is conserved off-shell for any vector field $\xi$, see equations (4) and (5). Then, by virtue of Poincaré lemma, we have introduced the Noether charge density (6), namely $K^{\mu \nu}(\xi)=\sqrt{-g} \tilde{K}^{\mu \nu}(\xi)$ where $\tilde{K}^{\mu \nu}(\xi)$ is given by Eq. (20). Now, we can define the near horizon conserved charge by integrating the Noether charge density over the event horizon

$\mathfrak{Q}(\chi)=\frac{1}{8 \pi G} \lim _{\rho \rightarrow 0} \int_{2-\text { sphere }}\left(d^{D-2} x\right)_{\mu \nu} \sqrt{-g} \nabla^{[\mu} \chi^{\nu]}$,

where we have replaced $\xi$ by $\chi$. By substituting Eqs. (44)(47) into the Eq. (48), we find that

$$
\begin{aligned}
\mathfrak{Q}\left(f, Y^{A}\right)= & \frac{1}{16 \pi G} \int_{\text {Horizon }} d^{2} x \sqrt{\operatorname{det} \Omega_{A B}} \\
& \times\left[2 \partial_{v} f+2 \kappa f-Y^{A} N_{A}\right] .
\end{aligned}
$$

In the stationary case, in which metric and $f$ are independent of the advanced time, Eq. (49) reduced to

$$
\begin{aligned}
\mathfrak{Q}\left(f, Y^{A}\right)= & \frac{1}{16 \pi G} \int_{\text {Horizon }} d^{2} x \sqrt{\operatorname{det} \Omega_{A B}} \\
& \times\left[2 \kappa f-Y^{A} N_{A}\right] .
\end{aligned}
$$

This result is exactly what has been found in Ref. [2], where the authors have considered the additional restrictions demanded by integrability (see Eq. (37) in Ref. [2]). As we have mentioned in the introduction, the near horizon geometry of non-extremal black hole solutions of a generally covariant theory of gravity exhibits an infinite-dimensional symmetry which is not exactly $\mathrm{BMS}_{4}$. The authors of [2] have shown that the full symmetry algebra includes two sets of supertranslations in semi-direct sum with two mutually commuting copies of Virasoro algebras. So, at the horizon we have found the so-called $\mathrm{BMS}_{4}^{H}$ algebra provided in Ref. [2].

\section{Conclusion}

We have considered a generally covariant theory of gravity, and found an off-shell conserved current Eq. (5) by virtue of the Bianchi identities. In order to define an extended off-shell ADT conserved current, we took variation of offshell conserved current Eq. (5) with respect to the dynamical fields. The generalized off-shell ADT current Eq. (11) is conserved for the asymptotically field-independent Killing vector fields and field-independent Killing vector fields admitted by spacetime everywhere. We have extended the generalized off-shell ADT current by replacing the Lee-Wald symplectic current by the symplectic current Eq. (10) to define the extended off-shell ADT current Eq. (12) which is conserved for asymptotically field-dependent Killing vector fields and field-dependent Killing vector fields admitted by spactime everywhere as well as field-independent one. Using the extended off-shell ADT current Eq. (12) we have defined extended off-shell ADT charge Eq. (15). Also, we have defined the conserved charge perturbation Eq. (16) by integrating of the extended off-shell ADT charge Eq. (15) over a spacelike codimension two surface. Then we applied the presented formalism to find the conserved charge perturbation of an asymptotically flat spacetime, which is presented in Ref. [25]. The obtained result Eq. (43) is exactly matched with the result of Ref. [1]. Then we have considered the fall-off conditions Eq. (44) near to the horizon of a non-extremal black hole. The considered fall-off conditions Eq. (44) are preserved under variation of the metric due to a diffeomorphism generated by the vector field Eq. (47). We have found near horizon conserved charges of the considered fall-off conditions Eq. (44) associated to the vector field Eq. (47) as the Noether charges.

Acknowledgements M. R. Setare thanks Dr. A. Sorouri for his help in improvement the English of the text.

Open Access This article is distributed under the terms of the Creative Commons Attribution 4.0 International License (http://creativecomm ons.org/licenses/by/4.0/), which permits unrestricted use, distribution, and reproduction in any medium, provided you give appropriate credit to the original author(s) and the source, provide a link to the Creative Commons license, and indicate if changes were made. Funded by SCOAP $^{3}$.

\section{References}

1. G. Barnich, C. Troessaert, JHEP 12, 105 (2011) 
2. L. Donnay, G. Giribet, H. A. Gonzlez, M. Pino (2016). arXiv:1607.05703 [hep-th]

3. H. Bondi, M.G.J. van der Burg, A.W.K. Metzner, R. Soc. Lond. Proc. Ser. A 269, 21-52 (1962)

4. R.K. Sachs, Proc. R. Soc. Lond. Ser. A 270, 103-126 (1962)

5. R.K. Sachs, Phys. Rev. 128, 2851 (1962)

6. G. Barnich, G. Compere, Class. Quant. Grav. 24, F15 (2007). Corrigendum: ibid 24 (2007) 3139

7. G. Barnich, C. Troessaert, Proceedings of the Workshop on Non Commutative Field Theory and Gravity, September 8-12 (2010)

8. S. Hollands, A. Ishibashi, J. Math. Phys. 46, 022503 (2005)

9. K. Tanabe, N. Tanahashi, T. Shiromizu, J. Math. Phys. 51, 062502 (2010)

10. A. Ashtekar, Phys. Rev. Lett. 46, 573 (1981)

11. A. Ashtekar, Asymptotic Quantization: Based on 1984 Naples Lectures, Monographs and Textbooks in Physical Science, 2. Bibliopolis, Naples (1987)

12. L. Donnay, G. Giribet, H.A. Gonzalez, M. Pino, Phys. Rev. Lett. 116, 091101 (2016)

13. R.G. Cai, S.M. Ruan, Y.L. Zhang, JHEP 09, 163 (2016)

14. M.R. Setare, H. Adami, Phys. Lett. B 760, 411 (2016)

15. M.R. Setare, Nucl. Phys. B 898, 259 (2015)

16. A. Strominger, JHEP 07, 152 (2014)

17. L. Susskind, A.I.P. Conf. Proc. 493, 98 (1999)

18. J. Polchinski, arXiv:hep-th/9901076 [hep-th]

19. J. de Boer, S.N. Solodukhin, Nucl. Phys. B 665, 545 (2003)

20. S. N. Solodukhin, arXiv:hep-th/0405252

21. M. Gary, S.B. Giddings, Phys. Rev. D 80, 046008 (2009)

22. G. Arcioni, C. Dappiaggi, Class. Quant. Grav. 21, 5655 (2004)

23. G. Arcioni, C. Dappiaggi, Nucl. Phys. B 674, 553 (2003)

24. G. Barnich, C. Troessaert, Phys. Rev. Lett. 105, 111103 (2010)

25. G. Barnich, C. Troessaert, JHEP 05, 062 (2010)

26. G. Barnich, C. Troessaert, JHEP 1311, 003 (2013)
27. G. Barnich, A. Gomberoff, H.A. Gonzalez, Phys. Rev. D 86, 024020 (2012)

28. G. Barnich, A. Gomberoff, H.A. Gonzalez, Phys. Rev. D 87, 124032 (2013)

29. G. Barnich, A. Gomberoff, H.A. Gonzalez, JHEP 05, 016 (2013)

30. G. Barnich, C. Troessaert, JHEP 03, 167 (2016)

31. B. Oblak, arXiv: 1610.08526 [hep-th]

32. G. Barnich, L. Donnay, J. Matulich, R. Troncoso, arXiv: 1510.08824 [hep-th]

33. G. Barnich, B. Oblak, JHEP 1406, 129 (2014)

34. G. Barnich, B. Oblak, JHEP 1503, 033 (2015)

35. G. Barnich, F. Brandt, Nucl. Phys. B 633, 3 (2002)

36. G. Barnich, Class. Quant. Grav. 20, 3685 (2003)

37. G. Barnich, G. Compere, J. Math. Phys. 49, 042901 (2008)

38. I. Fujisawa, R. Nakayama, Phys. Rev. D 91, 126005 (2015)

39. E.E. Flanagan, D.A. Nichols, arXiv: 1510.03386 [hep-th]

40. L.F. Abbott, S. Deser, Nucl. Phys. B 195, 76 (1982)

41. S. Deser, B. Tekin, Phys. Rev. Lett. 89, 101101 (2002)

42. S. Deser, B. Tekin, Phys. Rev. D 67, 084009 (2003)

43. A. Bouchareb, G. Clement, Class. Quant. Grav. 24, 5581 (2007)

44. S. Nam, J.-D. Park, S.-H. Yi, Phys. Rev. D 82, 124049 (2010)

45. W. Kim, S. Kulkarni, S.H. Yi, Phys. Rev. Lett. 111, 081101 (2013)

46. M.R. Setare, H. Adami, Eur. Phys. J. C 76, 187 (2016)

47. M.R. Setare, H. Adami, Nucl. Phys. B 902, 115 (2016)

48. M.R. Setare, H. Adami, Nucl. Phys. B 909, 345 (2016)

49. K. Prabhu, arXiv: $1511.00388 \mathrm{v} 2$ [gr-qc]

50. R.M. Wald, A. Zoupas, Phys. Rev. D 61, 084027 (2000)

51. J. Lee, R.M. Wald, J. Math. Phys. 31, 725 (1990)

52. R.M. Wald, Phys. Rev. D 48, 3427 (1993)

53. V. Iyer, R.M. Wald, Phys. Rev. D 50, 846 (1994)

54. S. Hyun, S.A. Park, S.H. Yi, JHEP 1406, 151 (2014)

55. L.A. Tamburino, J.H. Winicour, Phys. Rev. 150, 1039 (1966)

56. I. Booth, Phys. Rev. D 87, 024008 (2013) 